\title{
APORTACIONES AL ESTUDIO DE MEDIOS SALINOS. Prehistoria y Periodo Colonial en MéXico.
}

\section{CONTRIBUTIONS TO THE STUDY OF SALINE ENVIRONMENTS. Prehistory and Colonial Period in Mexico. \\ JOSÉ ANTONIO RUIZ GIL \\ jantonio.ruiz@,uca.es \\ UNIVERSIDAD DE CÁDIZ ${ }^{1}$}

[RECIBIDO: 07/02/ 2019; ACEPTADO: 07/04/2019]

http://dx.doi.org/10.25267/Riparia.2019.v5.02

\section{RESUMEN}

El axioma de partida es considerar la sal como un recurso estratégico de vital importancia para el Género Humano. Así pues, cualquier cultura, y en el caso concreto hablamos de las culturas mesoamericanas durante la época prehispánica, podía ser objeto de atención, máxime si se puede establecer un punto de comparación en el periodo colonial. Me interesa remarcar la posibilidad de establecer comparaciones culturales tanto en la tecnología como en los objetos propios de ella, como muestra de la capacidad del Ser Humano de encontrar soluciones similares o diferentes para idénticos problemas. En el caso concreto que propongo, la sal fue importante en Mesoamérica por la sencilla razón de que las localidades productoras estaban distribuidas de manera no uniforme sobre el paisaje, y muchas poblaciones sedentarias de gran tamaño no tenían un fácil acceso a pesar de necesitarla. Como consecuencia, se desarrollaron redes de comercialización de la sal desde tiempos prehispánicos.

Palabras Claves: Prehistoria, sal, periodo colonial, México.

1 Profesor Contratado Doctor. Área de Prehistoria. Universidad de Cádiz. Facultad de Filosofía y Letras, Avda. Gómez Ulla s/n, 11003 Cádiz.

J.A. Ruiz Gil, "Aportaciones al estudio de medios salinos. Prehistoria y Periodo Colonial en México”, RIPARIA 5 (2019), 31-56. 
J. A. RUIZ GIL

\begin{abstract}
The starting point is salt like strategic and life resource for Humankind. This sentence is very important if we can a comparison with de colonial period. Then, it is focusing in every culture and in this case the Mesoamerican prehispanic cultures. It's interesting remarking the possibility for cultural comparisons, as in technology as in the objects create for Culture, it showed the humankind skills for different or similar solutions searching for equal problems. In the case I proposed, salt was an important input in Mesoamerica by production places was distributed ununiformed way at the landscape simply. For this, nets of salt's markets were developed since prehispanic times.
\end{abstract}

KEY WORDS: Prehistory, salt, colonial period, Mexico.

"Aportaciones al estudio de medios salinos. Prehistoria y Periodo Colonial..." 


\section{Introducción}

Aunque el espacio geográfico que propongo sea Mesoamérica, quiero comenzar aclarando que la información de la que disponemos a día de hoy no es tan extensa como podría parecer. De hecho, dado que la bibliografía mexicana a pena s se utiliza cuando no se desconoce en trabajos realizados en España, es una de mis primeras intenciones dar a conocer un elenco básico. Desde un punto de vista bibliográfico voy a dar comienzo con el trabajo de síntesis La Industria salinera en México, de Úrsula Ewald (1994). Cierto es que ya existía algún trabajo local sobre la sal, como el de Miguel Othon de Mendizábal que desgraciadamente no he podido consultar directamente (Influencia de la sal en la distribución geográfica de los grupos indígenas de México [1928] 1946²; y que arqueólogos, como Anthony P. Andrews habían tocado el tema en el área Maya (1983). Posteriormente, etnoarqueólogos como Eduardo Williams (2003), e historiadores, como Laura Machuca (2007), han dado a las Humanidades la oportunidad de estudiar la sal mexicana para darle una correcta y actualizada valoración.

Ewald, además, fue la introductora del trabajo ${ }^{3}$ coordinado por Juan Carlos Reyes en 1995. La autora alemana nos entronca la industria salinera mexicana con el conocimiento europeo, partiendo de la obra Das Salz. (Leipzig, 1815) de Matthias.J. Schleiden; siguiendo con Le rôle du sel dans l'histoire (Paris, PUF, 1968) de Michel Mollat du Jourdin; Le sel et le fortune de Venise (Paris, 1978 y 1979, en dos vols.), y Le sel et le pouvoir. De l'an mil à la Rèvolution française (Paris, 1985), ambas de J.Cl. Hocqet; para terminar con el libro de S.A.M. Adshead Salt and civilization

2 Citado en L. MachuCA, Comercio de sal y redes de poder en Tehuantepec durante la época colonial. Centro de Investigaciones y Estudios Superiores en Antropología Social. México, 2007, 33.

${ }^{3}$ U. Ewald, Algunos apuntes y visiones en torno a la sal en México, J. C. REYES, La sal en México. Estudios sobre la sal, Colima, 1995. 
(London, 1992; recientemente reeditado por Palgrave MacMillan). En esta síntesis que realiza Ewald para los años noventa incluye también la publicación periódica Journal of salt-history: review of the International Commission for the History of Salt (CIHS) $=$ Annales d'histoire du sel, iniciada en 1993.

Además, la actividad salinera tradicional ha estado presente hasta mediados del siglo $\mathrm{XX}^{4}$, lo que actúa como una máscara ocultando, a diferencia de Europa, las evidencias más antiguas, principalmente las prehispánicas. Por tanto, muchos son los restos, pero mal conocidos y poco definidos. Las metodologías de estudio son principalmente históricas, etnográficas, y arqueológicas. El trabajo del arqueólogo Eduardo Williams (2001) representa el mejor ejemplo de estudio etnoarqueológico, basándose en el área costera de Michoacán (Williams, 2002), y en el lago Cuitzeo (Williams, 1999), recogiendo los usos tradicionales que han perdurado hasta la actualidad.

Hay que clarificar que, aunque hay muchos lugares citados, sólo las cuencas interiores del Eje Neovolcánico (o Sistema Volcánico Transversal) mexicano van a permitir un acercamiento más minucioso en época prehispánica; y la cuenca de la Meseta Mexicana ${ }^{5}$ en el período colonial. Hay casos específicos, como el de San Andrés de Tuxla, en Veracruz. Aquí, "El Salado", que es como se conoce esta salina de sal cocida de manantial, se encuentra tierra adentro ${ }^{6}$.

4 U. Ewald, La industria salinera en México. Fondo de Cultura Económica, 1994, 98.

5 A.F. Nieto, S.A. Alaniz, A. Camprubí, La Mesa Central de México: estratigrafía, estructura y evolución tectónica cenozoica. Boletín de la Sociedad Geológica Mexicana. Volumen Conmemorativo del Centenario. Temas Selectos de la Geología Mexicana, 2005, Tomo LVII, núm. 3, 285-318.

6 J.F. CEjA, Ixtlahuehue, la "Salina Vieja" de los Tuxlas, Revista Mexicana de Estudios Antropológicos, t-XXVIII, 41-47, Sociedad Mexicana de Antropología, 1982, México.

"Aportaciones al estudio de medios salinos. Prehistoria y Periodo Colonial..." 
Por ejemplo, en el Suroeste de México y norte del estado de Guerrero, las salinas inspeccionadas en el Procedimiento realizado en 1933 fueron atribuidas sin fundamento al periodo prehispánico ${ }^{7}$. En Tehuacán y en Oaxaca, entre los montículos de desechos, hay lajas de piedra que pueden ser tanto restos de los cristalizadores, como de los contenedores de salmuera o de los depósitos de lavado, incluso depósitos de agua para producir cerámica a gran escala ${ }^{8}$. Citar en este sentido que en Tehuacán hay cristalizadores de mampostería y enlucido interior que miden entre 2 por 3 y 6 por 8 metros. En el caso de Lambityeco, estado de Oaxaca, destaca el uso de la energía gravitatoria. De hecho, aunque no se descarte como posibilidad la sal cocida, el procedimiento conocido era por evaporación solar $y$, actualmente, en terrazas9. La evaporación solar es la técnica normal en las Salinas de Zapotlán, Puebla ${ }^{10}$; en las numerosas salinas del Pacífico de la costa de Michoacán ${ }^{11}$ y de Colima ${ }^{12}$; y en el Caribe, en Yucatán, al menos desde el Formativo Tardío (300 B.C.-300 A. D.) ${ }^{13}$. Por el contrario, la mayor relevancia histórica residió en las salinas de la costa. En el caso del estado occidental

7 J. Hernández Rivero, La producción de sal en Oaxaca. Una tradición milenaria. En J. C. Reyes, La sal en México..., 75.

8 J.R Parsons, The Last Saltmakers of Nesquipayac, México. An Archaeological Ethnography. Anthropological Papers. Museum of Anthropology, Univ. Michigan. no 92, 2001. Ann Arbor, Michigan, 267.

${ }^{9}$ C. VirAmonTES, La producción de sal en Oaxaca. Una tradición milenaria, J. C. REYES (COORD.), La sal en México...

10 R. MArtíneZ, B.R. Castellón, Zapotlán Salinas, Puebla. Una antigua comunidad de tradición salinera, J.C. REYes, La sal en México...; B.R. CAstellón, Entre cactus y barrancas: constructores de terrazas y productores de sal en el sur de Puebla, B.R. CASTELLón (Coord.) Sal y salinas: un gusto ancestral. Diario de Campo, Suplemento 51, Coord. Nacional del INAH, México, 105-115.

11 E. Williams Martínez, Producción y comercio de la sal desde el Michoacán antiguo, B.R. CASTELLÓN (Coord.) Sal y salinas...

12 J.C. REYES (Coord.), La sal en México...

13 E. Williams Martínez, Salt Production and Trade in Ancient Mesoamérica, J. Staller, M. Carrasco (Eds.), Pre-Columbian Foodways. Interdisciplinary Approaches to Food, Culture, and Markets in Ancient Mesoamerica, 2010, Springer, 175-190. 
méxicano de Colima, destacan las de Cuyutlán, estudiadas por Phil C. Weigand en 1997, como se incluye en la bibliografía.

\section{Periodo Prehispánico}

La producción de sal prehispánica es "invisible" a no ser por dos características: la localización próxima a una fuente de sal, y la presencia de montículos irregulares cubiertos de uno o dos tipos de cerámica, de baja calidad y muy comunes ${ }^{14}$. Aún así, no es posible a día de hoy separar fácilmente la cerámica salinera de la producción de sal misma, o diferenciar entre terrazas de uso agrícola o salinero ${ }^{15}$.

Con respecto a las implicaciones sociopolíticas y socioeconómicas de la fabricación de sal prehispánica, parece clara una generalización: que que hubo en algunas regiones un cambio de escala en la intensidad y en la tecnología productiva. Por ejemplo, entre el Postclásico Inicial y Medio del Valle de México con los aztecas del Postclásico Tardío, y entre el Clásico y el Epiclásico en la región Maya ${ }^{16}$.

Eduardo Williams se refiere a la sal como uno de los recursos estratégicos más importantes en Mesoamérica, y apunta a la cultura Olmeca (en torno al 1200 B.C.), aunque hipotetiza sobre la visita a los manantiales salinos unos mil años antes. Para este autor, en el Formativo Medio (ca. 900-300 B.C.) es cuando podemos situar el uso de vasos cerámicos para cocer ${ }^{17}$. En esta línea de argumentación, en la "Salina Vieja" de los Tuxlas (Veracruz), la cerámica corresponde al Preclásico Inferior, y la que el autor asocia con la sal corresponde a ollas grandes, o chalcaxitl, de cuello sencillo, y tecomates de fondo plano y

\footnotetext{
14 J.R PARSONS, The Last Saltmakers..., 276.

15 J.R PARSONS, The Last Saltmakers..., 277.

16 J.R PARsons, The Last Saltmakers..., 277.

17 E. Williams, Salt Production...
}

"Aportaciones al estudio de medios salinos. Prehistoria y Periodo Colonial..." 
paredes rectas divergentes ${ }^{18}$. Según Ceja Tenorio, el trabajo era estacional $\mathrm{y}$, tomando datos de principios del siglo $\mathrm{XX}$, para obtener un kilo de sal se necesitaba cocer 50 litros de salmuera. El pan de sal obtenido tenía forma de 'ladrillo'19.

Como ya he preludiado, me centraré en los estudios realizados en tres cuencas situadas en el Eje Neovolcánico: la ${\text { cuenca de } \text { México }^{20} \text {; el lago Cuitzeo }}^{21}$, y la cuenca de Sayula ${ }^{22}$.

En las depresiones del Lago Texcoco (que cubría Tenochtitlán excepto por el Este), y de los lagos Xaltocán y Zumpango, una franja de 500-1000 m. de grandes montículos de tierra cubiertos con cerámica TFM (Texcoco Fabric Marked, “impresiones de textil"), junto a caseríos y aldeas ${ }^{23}$, diversos autores se han referido a que los aztecas elaboraban la sal de estos lagos evaporando por cocción la salmuera en vasijas de barro ${ }^{24}$.

Según el fraile franciscano Bernardino de Sahagún, se tomaba la tierra con salitre, que llamaba tequixquitlalli ${ }^{25}$, se introducía en una tinaja, y se removía y colaba, para hacer panes de sal, restando residuos ${ }^{26}$. La invención de la sal se atribuía a la

18 J.F. CEJA, Ixtlahuehue... 2, figs. 2 y 3.

19 J.F. CEJA, Ixtlahuehue...

20 San Cristóbal de Nexquipayac, lago Texcoco, J.R. PARSONS, 1994; The Last Saltmakers..., citado en E. Williams MARTínEZ, Salt Production... .

${ }^{21}$ Idem nota 15.

22 C. Liot, Les salines prehispaniques du bassin de Sayula (Occident de Mexique): Milieu et techniques, BAR Int. Series 849. Oxford, 2000; citado en E. Williams, Salt Production....

23 E. Williams MartíneZ, La Sal de la tierra: Etnoarqueología de la producción salinera del Occidente de México. Zamora, Michoacán. El Colegio de Michoacán y la Secretaria de Cultura del Estado de Jalisco 34. 2003.

24 E. Williams MartíneZ, La Sal de ..., 31.

25 Fray Bernardino de Sahagún, Historia General de las Cosas de la Nueva España, 2 vols. Edición Juan Carlos Temprano. Dastin, S.L, 2001, 1046.

${ }^{26}$ Fray Bernardino de SAHagún, Historia General ..., 806. 
diosa de la sal Uixtociotl [Huixtocíhuatl] ${ }^{27}$, que se celebraba el primer día del mes séptimo Tecuilhuitontli, donde se sacrificaba una mujer ${ }^{28}$ y los sacerdotes tocaban muchas cornetas y caracolas $^{29}$.

Esta actividad salinera ha perdurado hasta el siglo XX en la población de Nexquipayac ${ }^{30}$. El proceso productivo fue descrito por J. Parsons en $1967^{31}$. Aquí, Williams diferencia los talleres de los de especialistas y de funcionamiento prolongado (básicamente hablamos de cocción); de los talleres mal definidos, efímeros y de época de secas (los de evaporación); si bien admite un tercer caso, que combina los dos anteriores ${ }^{32}$. Esta información etnográfica ha sido contrastada arqueológicamente en unas excavaciones de salvamento efectuadas en $1977^{33}$.

Los diversos autores que han tratado sobre la producción salinera mexicana han diferenciado dos tipos de montículos o tlateles (saladeras): los de tierra (producidos por los desechos del lavado del tequesquite) y los de tiestos o "tepalcateras" ${ }^{34}$. En ellos, más del $90 \%$ de las cerámicas son del tipo $\mathrm{TFM}^{35}$. Aunque Parsons en 2001 indicó que podían darse comportamientos diferenciados $^{36}$, lo característico son los amontonamientos irregulares de tierra; que en el caso de El Tepalcate (datado en el período Formativo Terminal), alcanzan una altura de ocho metros por 50 , y a lo sumo dos tipos de cerámica ${ }^{37}$.

${ }^{27}$ Fray Bernardino de SaHagún, Historia General ..., 175.

${ }^{28}$ Fray Bernardino de SaHagún, Historia General ..., 121.

29 Fray Bernardino de Sahagún, Historia General..., 177.

30 Descrita por Oscar Apenes en 1944, citado en J.R. PARSONS, The Last Saltmakers...

31 E. Williams MartíneZ, La Sal de ..., 40.

32 E. Williams MartíneZ, La Sal de ..., 43.

33 E. Williams MartíneZ, La Sal de ..., 38.

34 E. Williams Martínez, La Sal de ..., 116.

${ }^{35}$ E. Williams MartíneZ, La Sal de..., 36.

${ }^{36}$ J.R PARSONS, The Last Saltmakers..., 268.

${ }^{37}$ J.R PARSONS, The Last Saltmakers..., 265 y 267.

"Aportaciones al estudio de medios salinos. Prehistoria y Periodo Colonial..." 
Pasemos a la Cuenca de Sayula, a unos $60 \mathrm{~km}$. al sur de Guadalajara, Jalisco, y se extiende a lo largo de 30 kilómetros, y a lo ancho de $10^{38}$. De noviembre a mayo es seco, presentándose en verano las precipitaciones, momento de sedimentación lacustre y disolución de sales ${ }^{39}$. Cuando aumenta el índice de evaporación las sales solubles se concentran en los sedimentos superficiales, formando el conocido salitre o tequesquite ${ }^{40}$.

En la metodología de análisis que diseñó Liot en Sayula, era el proceso de sedimentación el punto de partida para el conocimiento de la producción de sal. Por un lado consideraba procesos naturales; por el otro, procesos antrópicos del tipo $\operatorname{artefactos}^{41}$. Las fuentes históricas comienzan con Alonso Ponce $^{42}$, y nos hablan de una cadena operativa que comenzaba con la recuperación del salitre, vertido posteriormente en tinajones, donde se añadía de agua y se removía para obtener una salmuera o lejía. Paralelamente, se había excavado un hoyo de menos de una vara, donde se colocaban un conjunto de ollas trabadas entre sí, luego la sal cristalizaba en los recipientes citados.

Para el siglo XIX, a finales concretamente, el explorador Carl Lumholtz visitó este paraje. Lumholtz cita concretamente el sitio de Cerro Colorado, que luego detallaré, sobre el que dice que se trata de un montículo formado por una aglomeración de

\footnotetext{
38 C. LIOT, Evidencias arqueológicas de producción de sal en la cuenca de Sayula (Jalisco): relación con el medio físico, estudio de tecnología, J. C. REYES, La sal en México..., 7-8.

${ }^{39}$ C. LiOT, Evidencias arqueológicas..., 8.

${ }^{40}$ C. Liot, Evidencias arqueológicas..., 9.

${ }^{41}$ C. Liot, Evidencias arqueológicas..., 5.

42 Alonso Ponce, Relación Breve y Verdadera de algunas cosas que sucedieron al padre Fray Bernardino de Sahagún. México, UNAM, 1973, 120-121; citado en C. LIOT, Evidencias arqueológicas..., 13.
} 
"coesillos" [cuicillo, templo de ídolos], de algo menos de 150 metros superficiales por 8 metros de alto, y hasta 1'5 metros de altura $^{43}$. En otro lugar, que él denomina Reparo, y que no resulta fácil ubicarlo hoy día, llegó a extraer cuatro de las cuarenta vasijas que pudo ver ${ }^{44}$. En opinión de Parsons son iguales a otros de piedra y enlucido de cal encontrados en Tonatico (estado de México) de $160 \mathrm{~cm}$. de alto por 140 de diámetro ${ }^{45}$.

A partir de este momento la producción salinera de Sayula es conocida en la literatura arqueológica mexicana. Citar la excavación realizada por Isabel Kelly ${ }^{46}$. Algunas estructuras se delimitaban con tiestos verticales, otras solo con el color y la textura de la tierra. Esta observación también fue advertida por el antropólogo americano Sleight ${ }^{47}$. Parsons cita los "Sayula salt pans”, de $40 \mathrm{~cm}$. de diámetro y $15-20 \mathrm{~cm}$. de profundidad ${ }^{48}$.

Varios son los yacimientos conocidos en la depresión de Sayula. Cerritos Colorados toma su nombre justamente del color de los tiestos que forman los montículos o tepalcateras ${ }^{49}$. Se han excavado tres estructuras cilíndricas, cuyas paredes estaban formadas por fragmentos de cerámica ${ }^{50}$. Se cita la reutilización de

43 C. Lumholtz, (Trad. B. DÁvalos), El México desconocido. Cinco años de exploración entre las tribus de la Sierra Madre Occidental en la tierra Caliente de Tepic y Jalisco, y entre los tarascos de Michoacán. Tomo II. México, 1960,313.

${ }^{44}$ C. Lumholtz, El México desconocido..., 317.

${ }^{45}$ J.R PARSONS, The Last Saltmakers..., 268.

46 Isabel Kelly, informe no publicado de 1944, 40-41, citada en C. Liot, La sal de Sayula: cronología y papel en la organización del poblamiento prehispánico, R. ÁviLA ET ALII. El Occidente de México: arqueología, historia y medio ambiente, perspectivas regionales. Actas del IV Coloquio de Occidentalistas., Universidad de Guadalajara y ORSTOM. Guadalajara, México.1998.

47 Sleight 1965, 158-160; citado en E. Williams Martínez, La Sal de..., 181-182.

48 J.R PARsOns, The Last Saltmakers..., 272-273.

${ }^{49}$ C. Liot, Evidencias arqueológicas ..., 17; E. Williams MartíneZ, La sal de..., 184.

${ }^{50}$ C. Liot, Evidencias arqueológicas..., 23.

"Aportaciones al estudio de medios salinos. Prehistoria y Periodo Colonial..." 
ceniza para recubrimiento, como en Nexquipayac (Texcoco) ${ }^{51}$. Por ejemplo, en otra fosa de $1 \mathrm{~m}$. diámetro y $60 \mathrm{~cm}$. de altura, con paredes de tepalcates y revoco color negro. Otra estructura (variante de La Motita), deja un montículo de acumulación de cajetes color café y línea roja en el borde ${ }^{52}$. Sobre la lixiviación, no hay pruebas de si se produjo a la manera actual (filtrado) o de la decantación/ concentración descrita por Ponce. Para los procesos de cocción se piensa mejor en un recipiente grande y hondo o en una fosa excavada en el suelo ${ }^{53}$. Los depósitos de desecho lixiviados contenían muy pocas sales ${ }^{54}$.

El yacimiento denominado Carmelita se caracteriza por el hallazgo de molederas y metates fragmentados, conchas, huesos, cerámicas, e incluso, sepulturas que denotan una actividad eventual en relación con la producción de sal ${ }^{55}$. Catherine Liot cita una pila casi cilíndrica, de fondo convexo, medio metro de profundidad y un enlucido de $2 \mathrm{~cm}^{56}$. La Motita es una elevación de $300 \mathrm{~m}$. de largo por 20 de ancho, asociada a un camino elevado sobre el ras de la laguna. Fue investigado por Isabel Kelly en 1940, quien lo describió ${ }^{57}$. Al igual que Cerros Colorados, tuvo una intervención arqueológica entre 1994 y 1995, donde se evidenció un conjunto de estructuras alineadas ${ }^{58}$. Una de las fosas era cilíndrica y presentaba una comunicación entre el cilindro y una depresión en pendiente ${ }^{59}$. Todas las estructuras se

51 F. Valdez, F., C. Liot, O. Schöndube, Los recursos naturales y su uso en las cuencas lacustres del sur de Jalisco: el caso de Sayula, E. Williams, P. C. Weigand, (Eds.) Las cuencas del Occidente de México. Época prehispánica, 1996, 345.

52 Riehm, Prehispanic Salt Boiling, Antiquity 35, 1961, 181-191, citado en F. VAldez, C. LIOT, O. SCHÖNDUBE, Los recursos..., 343.

${ }^{53}$ F. VALDEZ, C. LIOT, O. SCHÖNDUBE, Los recursos ..., 344.

${ }^{54}$ C. LIOT, Evidencias arqueológicas ...

55 C. LiOT, Evidencias arqueológicas ..., 16-17.

${ }^{56}$ C. LiOT, Evidencias arqueológicas..., 23.

${ }^{57}$ Citada en C. LIOT, Evidencias arqueológicas..., 18.

${ }^{58}$ F. VAldeZ, C. Liot, O. SchÖndube, Los recursos ..., fig.7.

${ }^{59}$ F. VAldeZ, C. LiOT, O. SCHÖNdube, Los recursos ..., fig. 9. 
encontraron rellenas de sedimento fino lagunar, y se han interpretado como de "filtración y decantación" 60 . Cerca de las citadas, se hallaron 4 fosas cilíndricas de 1 metro de diámetro por 15 centímetros de altura. Tenían una característica depresión en el fondo, de ahí que las consideraran "recipiente de decantación" 61 . Las paredes contaban con un recubrimiento de tepalcates y un enlucido de arcilla gris; dos se rellenaron con tepalcates, y las otras con sedimento fino de arcilla y arena ${ }^{62}$. El último tipo de fosa excavado era más bien un hoyo amorfo de unos $50 \mathrm{~cm}$. cuyas paredes irregulares "parecen ser hechas de cal"63. En el relleno, los tepalcates iban acompañados de ceniza y carbón, motivo por el que las consideraron "fogones" para cocer salmuera en vasijas ${ }^{64}$. Los autores plantean que fueran para cocer salmuera, o que sirvieran para decantar y concentrar antes de la cocción ${ }^{65}$.

Para finalizar con la metodología arqueológica, me referiré al yacimiento de San Juan, en Atoyac. Las estructuras descritas en San Juan abarcan seis tipos, desde las concentraciones de material cerámico $^{66}$ o tepalcateras; a los basureros o fosas de desechos, circulares u ovaladas ${ }^{67}$. También citan los $\operatorname{pozos}^{68}$ y los silos ${ }^{69}$.

\footnotetext{
${ }^{60} \mathrm{~F}$. VAldeZ, C. Liot, O. SCHÖNDUBE, Los recursos ..., 347.

${ }^{61}$ F. VAldeZ, C. LiOT, O. SCHÖNDUBe, Los recursos ..., fig. 9b.

${ }^{62}$ F. VAldeZ, C. Liot, O. SCHÖNDUBe, Los recursos ..., 348.

63 Idem, nota 55.

${ }^{64} \mathrm{~F}$. VAldeZ, C. LIOT, O. SCHÖNDUBE, Los recursos ..., 349.

${ }^{65}$ F. VAldeZ, C. Liot, O. SCHÖNDUBe, Los recursos ..., 348.

${ }^{66}$ F. VAldez, Las áreas domésticas en el sitio San Juan, Atoyac, Jalisco, E. Williams, Contribuciones a la arqueología y etnohistoria del Occidente de México. El Colegio de Michoacán. Zamora, México, 33, fig.6.

${ }^{67}$ F. VALDEZ, Las áreas domésticas..., 34, fig. 8.

${ }^{68}$ F. VALDEZ, Las áreas domésticas..., 34, fig. 9.

${ }^{69}$ F. VALDEZ, Las áreas domésticas..., 34, fig. 10.
}

"Aportaciones al estudio de medios salinos. Prehistoria y Periodo Colonial..." 
Terminan el repertorio los grandes recipientes cerámicos enterrados $^{70}$, similar a los de La Mota ${ }^{71}$.

\section{Cuenca del Cuitzeo}

Cada unidad productora de sal en el Cuitzeo, finca, de 50 $\mathrm{m}^{2}$, consiste de dos o más estiladeras, estructuras de madera que son usadas como filtros para separar la sal de la tierra por destilación. En cada finca hay varias canoas -contenedores de madera, hechos a manera de canoas alargadas- donde la salmuera que ha sido filtrada en la estiladera es evaporada por el sol ${ }^{72}$. Las herramientas usadas por los salineros son muy simples: palas, azadas y picos para excavar el suelo, carretillas para llevarlo a la estiladera, y cubetas para vaciar el agua en las canoas. Las herramientas empleadas en el pasado, sin embargo, eran muy diferentes: una especie de saco hecho de fibra de yute, era usado para llevar la tierra, y se usaban vasijas cerámicas para llevar el agua a la finca.

\section{La cerámica salinera}

La más conocida es la que presenta impresiones de tela. En algunos salares es la primera en utilizarse, no así en el valle de México, donde la precursora fue la alisada. Su uso más o menos continuado en Tehuacán durante 1300 años ha animado a Parsons a reflexionar sobre su utilidad. Para este autor la TFM se empleó en el secado final y empaquetado, considerando su fabricación a molde en hoyos forrados de tela, vegetal, madera o $\operatorname{arcilla}^{73}$.

70 C. Lumholtz, El México..., 317-321; F. Valdez, Las áreas domésticas..., 34, fig. 11;

C. Liot Evidencias arqueológicas ..., 20.

${ }^{71}$ C. LiOT, Evidencias arqueológicas ..., 21.

72 E. Williams MartíneZ, The Ethnoarchaeology of Salt Production at Lake Cuitzeo, Michoacan, Mexico. Latin American Antiquity, Vol. 10, 4, 1999, 400-414. E. Williams Martínez (Ed.), Bienes estratégicos del antiguo occidente de México. El Colegio de Michoacán. Zamora, México. 2004. E. WiLliams, Salt production....

73 J.R PARSONS, The Last Saltmakers ..., 279-280. 
En opinión de Williams ${ }^{74}$ posiblemente hubiera un componente teotihucano (del período Formativo) en estas cerámicas. Pero Parsons se inclina más por una adaptación al complejo Tepeyac del Postclásico que por una tradición ${ }^{75}$. No queda del todo clara, pues, la función, ya que se argumenta su producción local exclusiva para el empaquetado y transporte; mientras que Parsons intuye que la TFM jugó algún papel en el proceso final de cocción a baja temperatura, tal y como han interpretado otros autores ${ }^{76}$. En su argumentación, Parsons cita el caso de las Salinas de los Nueve Cerros, Guatemala ${ }^{77}$, datable entre el Formativo Terminal y el período Clásico Terminal mesoamericano. En esta salina se han definido tres tipos: unos cuencos hondos de base cóncava, moldes rotos intencionalmente por la base ${ }^{78}$; unas vateas, para el transporte de la salmuera del manantial a la salina; y unos cilindros altos y estrechos de fondo redondeado, moldes rotos deliberadamente ${ }^{79}$.

Estos vasos aparecen en una cantidad apreciable, tal y como sucede en salinas de la costa sur de Honduras, donde esta cerámica basta se asocia a grandes hogares-hoyo de forma rectangular y datación en el Postclásico ${ }^{80}$. Otro de los lugares que utiliza Parsons para fudamentar sus hipótesis es el Sureste de los Estados Unidos ${ }^{81}$. Se trata de una actividad de verano. Con una datación algo más antigua no asociada a una ocupación residencial, Brown distinguió tres estadios en la producción de sal, el antiguo de evaporación de la salmuera; uno de continuidad

${ }^{74}$ E. Williams MartíneZ, La Sal de ..., 35.

75 J.R PARSONS, The Last Saltmakers ..., 257.

${ }^{76}$ J.R PARSONS, The Last Saltmakers ..., 270.

77 B.K. S. Woodfill, B.D. Dillon, M. Wolf, C. Avendaño, R. Canter, Salinas de los Nueve Cerros, Guatemala: A mayor economic center in the southern Maya Lowlands. Latin America Antiquity 26(2), 2015, 162-169.

78 B.D. Dillon ET ALII 1988, 47, citado en J.R PARSONS, The Last Saltmakers ..., 271.

79 J.R PARSONS, The Last Saltmakers, 271.

${ }^{80} \mathrm{Idem}$.

81 BRown 1980, citado en J.R PARSONS, The Last Saltmakers..., 278.

"Aportaciones al estudio de medios salinos. Prehistoria y Periodo Colonial..." 
$\mathrm{y}$, finalmente, el de los cuencos calentados en hogares, tal y como era a la llegada de los europeos en los siglos XVI-XVII ${ }^{82}$. Brown distingue dos formas cerámicas ${ }^{83}$.

Sayula.

Como ya he adelantado uno de los elementos diagnósticos más relevantes es la evidencia cerámica para la producción de $\mathrm{sal}^{84}$. En algunos casos hay estructuras inmuebles de mampostería o de cerámica ${ }^{85}$. Se ha sugerido que hayan podido ser útiles para precipitar el carbonato de calcio menos soluble, mientras que el cloruro sódico permanecía. En Sayula las manchas blancas en la cara interna de algunos de los fragmentos cerámicos se han asociado a residuos de salitre ${ }^{86}$. Esta observación es difícil de llevar cuando también se ha escrito sobre la importancia de la industria lítica en el raspado de los tiestos. Por ejemplo, las hojas de obsidiana en Tehuacán, o en El Tepalcate ${ }^{87}$. Antes he hecho referencia a las excavaciones realizadas entre 1994 y 1995 en los yacimientos de Cerritos Colorados y La Motita. Ahora quiero comentar brevemente los periodos arqueológicos considerados:

- Fase Usmajac (300 a.C.-300 d.C.), sin indicios producción de sal;

- Fase Verdía (300-600 d.C.), donde aparece una tímida producción;

- Fase Sayula (600-1100 d.C.), con producción propia de una sociedad compleja, según los cánones antropológicos americanos; $\mathrm{y}$

82 J.R PARSONS, The Last Saltmakers..., 278.

${ }^{83}$ La información de este párrafo procede de J.R PARSONS, The Last Saltmakers..., 279.

84 A. Noyola 1994, 81, fig. 6 y 83, fig. 8h.

85 J.R PARSONS, The Last Saltmakers..., 268.

86 A. Noyola 1994: 64-5; Liot 1995, 19; E. Williams Martinez, La Sal de ..., 184.

${ }^{87}$ Idem nota 81. 
- Fase Amacueca (1100-1520 d.C.), en la que la producción era controlada desde fuera de la cuenca ${ }^{88}$.

En la Fase Sayula aparecen los 'cuencos salineros' ${ }^{89}$ y unos cuencos semiesféricos decorados con líneas rojas sobre el fondo beige ${ }^{90}$. La fase terminal de Isabel Kelly se llama Amacueca", donde los cuencos son sustituidos por "grandes cajetes de fondo plano y paredes rectas con el interior pulido y la base rugosa"92. Esa base rugosa puede corresponder a una impresión tipo TFM (a las que antes hice referencia), o a un revestimiento arcilla y paja $^{93}$. Para otros autores serían algo anteriores, concretamente de un período de transición entre el Sayula Tardío y el Amacueca Temprano ${ }^{94}$. En este caso con o sin borde-banda de pintura roja, y no apareciendo los cajetes de impresión de petate de San Juan, Atoyac; motivo por el que suponen una interpretación funcional (San Juan sería salinero) y otra cronológica (San Juan sería más reciente), opción en la que coincido con ellos ${ }^{95}$. Estas cerámicas rellenan las tres fosas cilíndricas mencionadas anteriormente. ${ }^{96}$ En la zona de Oaxaca, además de las citas sobre posibles hornos, encontramos

88 E. Williams MartíneZ, La Sal de ..., 185.

89 A. NoyolA, Análisis preliminar de la cerámica del fraccionamiento San Juan, Atoyac, Jalisco, E. Williams MARTíneZ, Contribuciones a la arqueología y etnohistoria del Occidente de México. El Colegio de Michoacán. Zamora, México, 1994, 55-91.

57; E. Williams MartíneZ, La Sal de ....

90 C. Liot, Evidencias arqueológicas...; E. Williams Martínez, La Sal de ..., 200, fig. 33.

91 Patricia Kelly, citada en A. NoyOLA, Análisis preliminar ..., 56.

92 C. Liot, Evidencias arqueológicas..., 19; A. NoyolA, Análisis preliminar ..., 64-5.

93 Valdez, citado en E. Williams MartíneZ, La Sal de ..., 201, fig. 34.

94 Ma.R. Acosta Nieva, J-P-Emphoux, S. Ramírez Urrea, El sur de la cuenca de Sayula, Jalisco: el sitio Caseta, un ejemplo, E. Williams, P.C. Weigand (Eds.), Las Cuencas del Occidente de México. Época Prehispánica. El Colegio de Michoacán, 1996, 378.

${ }^{95}$ Ma.R. Acosta, J-P-Emphoux, S. Ramírez, El sur ..., 382; ver también F. VAldeZ, C. LIOT, O. SCHÖNDUBE, Los recursos ..., 346.

${ }^{96}$ C. Liot, Evidencias arqueológicas ..., 20.

"Aportaciones al estudio de medios salinos. Prehistoria y Periodo Colonial..." 
abundantes restos de unas jarras del periodo posclásico con signos evidentes de haber sido sometidas al fuego ${ }^{97}$.

\section{Período Colonial}

La explotación de la sal en periodo colonial es una de las cuestiones más interesantes que he podido estudiar en México. Lo primero a destacar es que la Corona española fue permisiva con la producción doméstica, pero no así donde se podía producir en grandes cantidades ${ }^{98}$. En este sentido, la importancia estratégica de la sal (junto con el mercurio) para el "beneficio de patio", indispensable en la industria de la plata en el México colonial es un tema que no puede soslayarse, como seguídamente expondré.

La Real Cédula de 1591 imponía el monopolio de todas las salinas de América, pero quedaron fuera las salinas pertenecientes a las comunidades indígenas ${ }^{99}$. Por lo tanto, hay que indicar que, a nivel de producción de sal, el gran cambio que se va a producir, no es el de la incorporación de grandes explotaciones, que también, sino la difusión y el predominio de las técnicas indígenas de evaporación sobre las de cocción. Y así permaneció hasta finales del siglo XVIII, donde la presión sobre las salinas indígenas iba desde la intervención, como en Tehuantepec, la concesión de derechos a los colonos (Yucatán), la recuperación (recomendación en la Ordenanza de Intendentes de 1786), se recomendaba recuperar las salinas no explotadas por los indígenas. Desde 1770 se el cobro de una anualidad simbólica, o el propio arrendamiento ${ }^{100}$.

Esto supuso, no sólo un cierto florecimiento durante el siglo XVI, sino que fuera la sal la más importante producción

\footnotetext{
97 J.R PARSONS, The Last Saltmakers..., 278.

98 U. Ewald, La industria salinera...; E. Williams Martínez 2003, 14.

${ }^{99}$ L. MACHUCA, Comercio de sal ..., 118.

100 Idem, 118.
} 
económica de ámbito regional al estar asociada al cacicazgo. Este caso es el que estudia Laura Machuca en Tehuantepec. El cacique local buscaba el apoyo de las instituciones coloniales, ya fuera personalmente en el Alcalde Mayor, o en el Mayorazgo. Las salinas, pasaron a ser un bien enajenado a la comunidad indígena y, cuando murió la última heredera zapoteca, pasó a un grupo colateral de etnia huave. Para 1781 pasaron a la Administración Real (hasta la Independencia), contándose once salinas, 4 pertenecientes a los caciques, tres a los huaves y 4 a los chontales $^{101}$.

En segundo lugar, destacar que “...nadie intentó introducir adelantos tecnológicos ni experimentar en las salinas para producir un cloruro de sodio más puro..."102. Y la transferencia de tecnología era necesaria... al menos para la minería de la plata.

Las salinas de Santa María y del Peñol Blanco (mantengo la cita de Ruiz Medrano) eran lagunas estacionales, explotadas desde la segunda mitad del siglo XVI para la explotación minera de Zacatecas, Pachuca, Charcas, y Guanajuato. Del período prehispánico hay evidencias de explotación salina en Salinas de Hidalgo, y en La Quemada. En 1555, en la mina La Purísima, de Pachuca, Bartolomé Medina descubrió el modo de fundir plata de baja calidad. Este método se conoce como de "patio" o "cazo"103.

Peñón Gordo producía saltierra y un poco de sal en grano. Esto suponía un trabajo añadido de lixiviado de la saltierra o de disolución del grano evaporando la salmuera. Dependiendo de la humedad y de la temperatura las eflorescencias salinas

${ }^{101}$ L. MACHuCA, Comercio de sal ...,113 y 151.

102 U. EwALD, La industria salinera..., 94.

103 C.R. Ruiz Medrano, Las salinas de Santa María y del Peñol Blanco en la segunda mitad del siglo XVI. Frontera, mano de obra y articulación territorial, Tzintzun. Revista de Estudios Históricos 55, 2012, 82.

"Aportaciones al estudio de medios salinos. Prehistoria y Periodo Colonial..." 
aparecían en costras escamosas, la primera capa "(la barradura de la tez)" era la más apreciada por los mineros, pues a más profundidad más tierra y menos sal. Por lo tanto, mejor en el borde de la laguna que en el centro. Para obtener calidad era fundamental recoger en costras, no en polvo, por eso se requería una mano de obra habilidosa, indígena ${ }^{104}$. El área explotada en Peñón Blanco (aquí mantengo la cita de Ewald) era de 1 milla de largo x media de ancho ${ }^{105}$. Había otras salinas "filiales", de menor producción y de menor calidad (Santa Clara, Salitral de Carrera y El Morro, en grano, sal espumilla y tequesquite ${ }^{106}$.

\section{Discusión}

Para terminar, pensamos más interesante (dado el espacio que manejamos) establecer unos términos de discusión, mejor que dar unas conclusiones. En primer lugar, resaltar que la sal, como sucede con otros 'descubrimientos' de la Humanidad, no se ajusta a un patrón difusor. Al contrario, como Ifrah (2002, 24-25) apunta, hay un conocimiento compartido de especie que podríamos conocer como « estructural». Wlliams ${ }^{107}$ nos resume el proceso en cinco fases : en la primera se cosecha el tequesquite (salitre), posteriormente se amontona para ser lixiviado en un filtro de horquetas de troncos y base de palos, carrizos, zacate (una planta herbácea local) y arena. En tercer lugar, se ponen sobre el filtro capas de lodo o arcilla, sobre el que se deposita el salitre. Después, el lixiviado cae en la "taza", y se transporta a las "eras".

Así pues, la primera cuestión es la de la propia limitación de las fuentes, por ejemplo, Sahagún no cita la « pila », o sea, el elemento de lixiviar ${ }^{108}$. La Relación Geográfica de Coxcatlán

\footnotetext{
104 U. EWALD, La industria salinera... 94-95.

105 Idem, 96.

106 Idem, 97.

107 E. Williams Martínez, La Sal de ..., 177-178.

108 E. Williams MartíneZ, La Sal de ..., 42.
} 
(Puebla s. XVI) muestra unos estanques poco profundos rectangulares llamados "pilas de sal", en este caso para la evaporación. No hay evidencias para la costa de Michoacán de tinas de evaporación, como en la Sayula prehispánica ${ }^{109}$. La filtración de la salmuera se podía realizar en el siglo XVI según las Relaciones con un filtro sobre trípode en Cuzamala, o un colador sobre una vasija en Motines ${ }^{110}$. La aparición en Oaxaca (Lambityeco) de depósitos de carbonato cálcico, nos refiere el uso de cal en estos procesos, como ocurre actualmente en La Placita (Michoacán) ${ }^{111}$.

Estos cristalizadores pueden tener un origen indígena. Pero hay otro elemento al que sí se atribuye un origen autóctono : las canoas. En la Relación de Zapotitlan de 1579, Juan de Estrada habla de unas canoas a modo de artesas agujereadas que usan de tamiz con esteras, colocándose abajo unas ollas. Este argumento ha sido aducido por Reyes ${ }^{112}$ como una variante del tapextle. Las canoas se han estado usando tradicionalmente en Cuyutlán, Colima $^{113}$ y en la costa de Michoacán ${ }^{114}$.

De cualquier forma, la pieza para filtrar era el tapeixtle, llamado tapeite en la costa de Guerrero. Una vez filtrado se llevaba en cestas a las «eras », de unos 6x3 m., donde cada 18 eras forman un Plan. Cada cesta tiene una capacidad de 20 litros, y salen 2 o 3 cestas por día y era, unos 25/30 kg/día. En conjunto un Plan puede producir unas 7 tn. por campaña ${ }^{115}$. Por otra parte, resulta muy interesante la discusión sobre el origen del Tapextle.

\footnotetext{
109 E. Williams MartíneZ, La Sal de ..., 145.

${ }^{110}$ E. Williams Martínez, La Sal de ..., 168, fig. 32.

111 E. Williams MartíneZ, La Sal de..., 45.

112 J.C. REYES, El Tapextle salinero: notas sobre su origen, distribución y variantes. En Bienes estratégicos del antiguo occidente de México (E. Williams). El Colegio de Michoacán. Zamora, México, 2004, 189.

113 J. C. REYES (COORD.), La sal...

114 E. Williams Martinez, La Sal de ..., 2002 y 2004.

115 E. Williams MartineZ, Salt Production....

"Aportaciones al estudio de medios salinos. Prehistoria y Periodo Colonial..."
} 
Se desconoce su antigüedad en Mesoamérica, no hay datos arqueológicos, y en las fuentes del XVII no aparece mencionado. Pero, según Williams, las Relaciones geográficas del XVI documentan un tipo similar. Para otros autores se propone su uso primero en Oriente, a semejanza de "procedimientos acostumbrados en las salinas de China y Filipinas"), ya que solo se usa en la costa del Pacífico mexicano, y que la presencia de "indios chinos" se asocia al cultivo del cocotero desde el siglo XVII. Y, de hecho, ya a fines del siglo XIX, se conocían unos 'albornoces orientales' "hechas con tiras de hojas de palma superpuestas"116, originarias de la China (Manila). Es decir, que la relación colonial entre Filipinas y la costa pacífica mexicana era un hecho.

Con respecto a los cacharros cerámicos, la descripción de Ponce del siglo XVI no se corresponde con las encontradas arqueologicamente. En este sentido, la identificación de las vasijas utilizadas para la cocción de la sal es uno de los problemas del estudio arqueológico de tecnologías de producción de sal, sobre todo cuando no se encuentran en estructuras de combustión, ocultando posibles usos, características de la pasta (es curioso encontrar en la bibliografía opiniones favorables tanto a la bondad del bruñido interior como a lo favorable de la rugosidad interna), tamaño y forma (diferentes volúmenes que marcarían diferentes unidades de medida ${ }^{117}$. Williams se inclina por su uso como unidad de valor para el intercambio ${ }^{118}$.

En realidad estamos hablando de difentes vasijas, unas para transportar la salmuera, o de Maruata ${ }^{119}$. Otras para transportar agua para el lixiviado, "Chondas"120 en San Juan de

\footnotetext{
116 C. Lumholtz, El México ...,324, y foto p. 341.

117 C. Liot, Evidencias arqueológicas..., 26.

118 E. Williams MartíneZ, La Sal de ..., 38.

119 E. Williams Martínez, La Sal de ...,159, fig.31.

${ }^{120}$ E. Williams MartíneZ, La Sal de ..., 129, fig. 16.
} 
Zinapécuaro (Cuizeo). También, que son las más conocidas cerámicas salineras, las que se usan para la cocción o el secado. Sirven de molde para el pan de sal. Según unas fuentes hay que romperlo, ya sea porque la sal se adhiere a las paredes, o porque resulta bueno para la comercialización ${ }^{121}$; según otras se pueden usar varias veces. Si el transporte es de proximidad, hay autores que proponen el uso de cestas ${ }^{122}$.

Finalmente, como último elemento de discusión, a pesar de la fácil transferencia de tecnología que suponía labrar una nueva salina, ejemplo de Salinas de Araya- La Tortuga ${ }^{123}$, la autoridad colonial española prefirió utilizar la tecnología indígena a cambio de su mano de obra barata.

\section{Agradecimientos}

A El Colegio de Michoacán, Zamora de Hidalgo, México, donde en noviembre de 2013 tuve ocasión de realizar una estancia de investigación. Y a César Borja, organizador de la reunión del Grupo Andaluz de Cuaternario celebrada en Sevilla en 2016 sobre el tema de las salinas de interior, donde presenté una primera versión de este trabajo.

\footnotetext{
121 C. LiOT, Evidencias arqueológicas ..., 28.

122 J.R PARSONS, The Last Saltmakers..., 221.

123 A. ANTCZAK, K. ANTCZAK, M.M. ANTCZAK, Risky business: historical archaeology of the Dutch salt enterprise on La Tortuga Island, Venezuela (1624-38). Post-Medieval Archaeology 49/2, 2015, 189-219.
}

"Aportaciones al estudio de medios salinos. Prehistoria y Periodo Colonial..." 
RIPARIA VOL. 5 (2019)

\section{Bibliografía}

Ma.R Acosta Nieva,.J-P. EmphOux, S. Ramírez UrReA, El sur de la cuenca de Sayula, Jalisco: el sitio Caseta, un ejemplo; en Las Cuencas del Occidente de México. Época Prehispánica, E. Williams, P.C. WEIGAND (Eds.), El Colegio de Michoacán, 1996, pp. 367-393.

ALONSO PONCE, Relación Breve y Verdadera de algunas cosas que sucedieron al padre Fray Bernardo de Sahagún. México, 1973, UNAM.

Alonso Ponce, Viaje a Nueva España (Antología). Selección y prólogo de Andrés Henestrosa. Biblioteca Enciclopédica Popular 184, Secretaría de Educación Pública. México 1947.

A.P. Andrews, Ancient Maya Salt Production and Trade, Tucson, 1983, University of Arizona Press.

A. ANTCZAK, K. ANTCZAK, M.M. ANTCZAK, Risky business: historical archaeology of the Dutch salt enterprise on La Tortuga Island, Venezuela (1624-38). Post-Medieval Archaeology 49/2, 2015, 189-219.

J.F. CEjA TENORIO, Ixtlahuehue, la "Salina Vieja" de los Tuxlas. Revista Mexicana de Estudios Antropológicos, t-XXVIII, 41-47. Sociedad Mexicana de Antropología, México 1982.

B.R. CASTELLÓN HuERTA, Entre cactus y barrancas: constructores de terrazas y productores de sal en el sur de Puebla, B.R. CASTELLÓN HuERTA (Coord.) Sal y salinas: un gusto ancestral. Diario de Campo, Suplemento 51, Coord. Nacional del INAH, México 2008, pp. 105-115. CASTELlÓN HUERTA, BlAS R. (2014). La producción de panes de sal en el sur de Puebla. Arqueología Mexicana 21 (125), pp. 74-79.

U. EWALD, La industria salinera en México. Fondo de Cultura Económica, 1994.

U. EWALD, Algunos apuntes y visiones en torno a la sal en México, J.C. REYES, La sal en México, Colima, 1995, pp. xiii-xxiv.

J. HeRnÁndez Rivero, La producción de sal en Oaxaca. Una tradición milenaria. En La sal en México, J.C. REYES, La sal en México, Colima, 1995, pp. 75-112.

Fray Bernardino De SAHagún, Historia General de las Cosas de la Nueva España, 2 vols. Edición Juan Carlos Temprano. Dastin, S.L. 2001. G. IfRAH, Historia Universal de las Cifras, Ed. Espasa, 2002.

C. LIOT, Evidencias arqueológicas de producción de sal en la cuenca de Sayula (Jalisco): relación con el medio físico, estudio de tecnología, J.C. REYES, La sal en México, Colima, 1995, pp.1-31. 
C. LIOT, La sal de Sayula: cronología y papel en la organización del poblamiento prehispánico, R. ÁvILA (Ed.) El Occidente de México: arqueología, historia y medio ambiente, perspectivas regionales. Actas del IV Coloquio de Occidentalistas, Universidad de Guadalajara y ORSTOM. Guadalajara, México 1998.

C. LIOT, Les salines prehispaniques du bassin de Sayula (Occident de Mexique): Milien et techniques. BAR. I.S. 849, Oxford 2000.

C. LumHOlTZ, El México desconocido. Cinco años de exploración entre las tribus de la Sierra Madre Occidental en la tierra Caliente de Tepic y Jalisco, y entre los tarascos de Michoacán, B. Dávalos (Trad.) Tomo II. México 1960.

A.F. Nieto-Samaniego, S.A. Alaniz-Álvarez, A. Camprubí I CANO, La Mesa Central de México: estratigrafía, estructura y evolución tectónica cenozoica. Boletín de la Sociedad Geológica Mexicana. Volumen Conmemorativo del Centenario. Temas Selectos de la Geología Mexicana, 2005, tomo LVII, núm. 3, 285-318.

A. NOYOLA, Análisis preliminar de la cerámica del fraccionamiento San Juan, Atoyac, Jalisco, E. WiLliams (Ed.), Contribuciones a la arqueología y etnohistoria del Occidente de México, El Colegio de Michoacán, Zamora, México 1994, pp. 55-91.

L.Machuca Gallegos, Comercio de sal y redes de poder en Tehuantepec durante la época colonial, Centro de Investigaciones y Estudios Superiores en Antropología Social. México 2007.

R. Martínez Chilpa, B.R. CASTellón Huerta, Zapotlán Salinas, Puebla. Una antigua comunidad de tradición salinera, J.C. REYES (Coord.), La sal en México, Colima, México 1995, pp. 53-73.

J.R. PARSONS, The Last Saltmakers of Nesquipayac, México. An Archaeological Ethnography. Anthropological Papers. Museum of Anthropology, Univ. Michigan. No 92. Ann Arbor, Michigan 2001. J.C. REYES (Coord.), La sal en México, Colima, México 1995.

J.C. REYES, El Tapextle salinero: notas sobre su origen, distribución y variantes, E. Williams (Ed.), Bienes estratégicos del antiguo occidente de México, El Colegio de Michoacán. Zamora, México 2004, pp. 183-ss.

K. RIEHM, Prehispanic salt boiling, Antiquity 35, 1961, pp. 181-191.

C.R. Ruiz Medrano, Las salinas de Santa María y del Peñol Blanco en la segunda mitad del siglo XVI. Frontera, mano de obra y articulación territorial, Tzintzun. Revista de Estudios Históricos 55, 2012, pp. 75-ss.

O. SCHÖNDUBE, La región de Sayula vista a través de las fuentes etnohistóricas, E. Williams (Ed.), Contribuciones a la arqueología y

"Aportaciones al estudio de medios salinos. Prehistoria y Periodo Colonial..." 
etnohistoria del Occidente de México, El Colegio de Michoacán. Zamora, México 1994, pp. 325-339.

F. VALDEZ, Las áreas domésticas en el sitio San Juan, Atoyac, Jalisco, E. Williams (Ed.), Contribuciones a la arqueología y etnohistoria del Occidente de México, El Colegio de Michoacán. Zamora, México 1994, pp. 23-53. F. VALDEZ, C. LIOT, O. SCHÖNDUBE, Los recursos naturales y su uso en las cuencas lacustres del sur de Jalisco: el caso de Sayula, E. Williams, P.C. Weigand, (Eds.), Las cuencas del Occidente de México. Época prehispánica, México 1996, pp. 325 ss.

C. VirAmontes ANZuRES, La producción de sal en Oaxaca. Una tradición milenaria. J.C. REYES (Coord.), La sal en México, Colima, México 1995, pp. 33-51.

P.C. WEIGAND, Salinas y salineros: manufactura prehispánica de sal en el Occidente de México, Antropología en Jalisco: Una Visión Actual 9, 1997, pp. 5-23.

E. WiLliams MARTÍNEZ (Ed), Contribuciones a la arqueología y etnohistoria del Occidente de México, El Colegio de Michoacán, Zamora, México 1994. E. Williams MartíneZ, Cerámica salinera del occidente de México, E. Williams, PH. WeIGAND (eds.), Estudios cerámicos en el Occidente y Norte de México, El Colegio de Michoacán e Instituto Michoacano de Cultura 2001, pp. 175-218.

E. Williams MartíneZ (Ed), Bienes estratégicos del antiguo occidente de México, El Colegio de Michoacán, Zamora, México 2004.

E. Williams MartíneZ, P.C. WeIgand, (Eds.) Las cuencas del Occidente de México. Época prehispánica, 1996.

E. Williams MARTínEZ, The Ethnoarchaeology of Salt Production at Lake Cuitzeo, Michoacan, Mexico, Latin American Antiquity, Vol. 10, 4, 1999.

E. Williams MartíneZ, Salt Production in the Coastal Area of Michoacan, Mexico. An ethnoarchaeological study. Ancient Mesoamerica, 13, 2002, pp. 237-253.

E. WILLIAMS MARTÍNEZ, La Sal de la tierra: Etnoarqueología de la producción salinera del Occidente de México, Zamora, Michoacán. El Colegio de Michoacán y la Secretaria de Cultura del Estado de Jalisco, 2003.

E. Williams MartíneZ, Producción y comercio de la sal desde el Michoacán antiguo, B.R. CASTELlÓN HuERTA (Coord.) Saly salinas: un gusto ancestral. Diario de Campo, Suplemento 51, Coord. Nacional del INAH, México 2008, pp. 41-49. 
E. Williams MartíneZ, Salt Production and Trade in Ancient Mesoamérica, J. Staller, M. CARrasco, (eds.), Pre-Columbian Foodways. Interdisciplinary Approaches to Food, Culture, and Markets in Ancient Mesoamerica, Springer 2010, pp. 175-190.

B.K.S. WoOdfill, B.D. Dillon, M. Wolf, C. Avendaño; R. CANTER, Salinas de los Nueve Cerros, Guatemala: A mayor economic center in the Southern Maya Lowlands. Latin America Antiquity 26(2), 2015, pp. 162-169.

"Aportaciones al estudio de medios salinos. Prehistoria y Periodo Colonial..." 